\title{
Herniorrafía con la técnica de Lichtenstein versus Nyhus en el manejo de las hernias inguinales y sus complicaciones postoperatorias en un hospital del Perú
}

\author{
Hernia repair comparing Lichtenstein and Nyhus \\ techniques for the management of inguinal hernia and its \\ postoperative complications in a Peruvian hospital
}

Correspondencia

Antonio M. Quispe

drantonioquispe@gmail.com aquispe@jhu.edu

Recibido: 20/07/2016 Arbitrado por pares

Aprobado: 31/0/2016

Citar como: Granda AC, CorreaTineo S, Quispe AM. Herniorrafía con la técnica de Lichtenstein versus Nyhus en el manejo de las hernias inguinales y sus complicaciones postoperatorias en un hospital del Perú. Acta Med Peru. 2016;33(3):208-16
Ana C. Granda1', Santos Correa-Tineo 1,2, Antonio M. Quispe ${ }^{3,4}$

1 Facultad de Medicina, Universidad Nacional Mayor de San Marcos. Lima, Perú.

2 Servicio de Cirugía, Hospital Militar Central Luis Arias Schreiber. Lima, Perú.

3 Department of International Health, Johns Hopkins Bloomberg School of Public Health. Baltimore, EE.UU.

4 Grupo de Investigación Biomédica, Web Med Research. Lima, Perú.

\section{RESUMEN}

Objetivo: Determinar la incidencia de complicaciones tempranas y tardías según técnicas de Lichtenstein y Nyhus en el manejo de hernias inguinales en el Hospital Militar Central (HMC). Material y métodos: Transversalmente se analizaron todos los casos de hernia inguinal tratados quirúrgicamente en el HMC durante el período 2012-2014. Resultados: 202 casos $(65 \%$, varones) fueron analizados, de los cuales el $11 \%$ y el $89 \%$ fueron operados con las técnicas de Nyhus y Lichtenstein, respectivamente. Al comparar ambas técnicas no se encontraron diferencias en sus tiempos quirúrgicos $(43,0 \pm 13,6$ vs. 39, $1 \pm 8,9$ minutos; $p=0,140)$, tiempos de hospitalización $(3,9 \pm 1,2$ vs. $3,8 \pm 0,8$ días; $p=0,635)$ e incidencia de complicaciones tardías $(4 \%$ vs. $0 \%$; $p=0,603$ ), pero sí al comparar la incidencia de complicaciones tempranas ( $17 \%$ vs. $0 \%$; $\mathrm{p}=0.028)$. Las complicaciones tempranas y tardías más frecuentes fueron de tipo neuralgias ( $12 \%$ vs. $0 \%$ ) y recurrencias ( $3 \%$ vs. $0 \%$ ), respectivamente. Al análisis de regresión se encontró que tanto el tiempo quirúrgico (Odds ratio [OR]; 1,07; Intervalo de confianza [IC] al 95\%: 1,04-1,11) como el tiempo de hospitalización (OR: 1,55; IC 95\%: 1,10-2,19) se encontraban significativamente asociados la ocurrencia de complicaciones tempranas. Conclusiones: En la experiencia del HMC ambas técnicas, Lichtenstein y Nyhus, demostraron ser alternativas seguras en el manejo quirúrgico de hernias inguinales, siendo las complicaciones tempranas más frecuentes con la técnica de Lichtenstein y, en ambos casos, más frecuentes mientras mayor el tiempo quirúrgico y el tiempo de hospitalización.

Palabras clave: Hernia inguinal; Herniorrafía; Complicaciones posoperatorias; Estudio comparativo (fuente: Decs BIREME). 


\begin{abstract}
Objective: To determine the frequency of early and late complications using the Lichtenstein and Nyhus techniques for the management of inguinal hernias in the Peruvian Military Central Hospital. Material and methods: A cross-sectional study was performed, in which we analyzed the all gases of inguinal hernia that underwent surgical repair during the 2012-2014 period. Results: Two hundred and two cases were analyzed ( $65 \%$ from male subjects), of which $11 \%$ and $89 \%$ were operated using the Nyhus and Lichtenstein techniques, respectively. When comparing both techniques, no differences were found in terms of surgical time $(43.0 \pm 13.6$ vs. $39.1 \pm 8.9$ minutes; $p=0.140)$, hospitalization duration $(3.9 \pm 1.2$ vs. $3.8 \pm 0.8$ days; $p=0.635$ ), and late complications ( $4 \%$ vs. $0 \% ; p=0.603$ ), but we found significant differences in the frequency of early complications ( $17 \%$ vs. $0 \% ; p=0.028)$. The most frequent early and late complications were neuralgia $(12 \%$ vs. $0 \%)$ and recurrence ( $3 \%$ vs. $0 \%$ ), respectively. Using the logistic regression analysis we found that both the operating time (odds ratio [OR]: 1.07; $95 \%$ confidence interval [Cl]: 1.04-1.11) and the length of hospitalization (OR: 1.55; $95 \% \mathrm{Cl}: 1.10-2.19)$, were significantly associated with the occurrence of early complications. Conclusions: In the experience of the Peruvian Military Central Hospital both Lichtenstein and Nyhus techniques proved to be quite safe for the surgical management of inguinal hernias. Early complications were more frequently observed with the Lichtenstein technique; and, with both techniques, complications were more frequent with longer surgical times and prolonged hospitalization times.
\end{abstract}

Key words: Hernia, inguinal; Herniorrhaphy; Postoperative complications; Comparative study (source: MeSH NLM).

\section{INTRODUCCIÓN}

La cirugía de hernia inguinal continúa siendo uno de los procedimientos quirúrgicos más frecuentemente practicados en los hospitales generales a nivel mundial ${ }^{[1]}$. A pesar de ello, no existe un consenso entre los cirujanos con respecto a cuál de las muchas técnicas quirúrgicas disponibles podría considerarse como la técnica quirúrgica de elección para el manejo de las hernias inguinales en general. Esto se debe a que mientras un meta-análisis sugería la superioridad del abordaje laparoscópico por sobre el abordaje abierto ${ }^{[2]}$, otro reportó que cuando ambos abordajes incluían la colocación de una malla, el abordaje abierto resultaba ser superior al abordaje laparoscópico ${ }^{[3]}$. Esto se debe a que si bien la herniorrafía laparoscópica ofrece como beneficios una menor incidencia de seromas o hematomas ${ }^{[4]}$, adormecimiento ${ }^{[5]}$ y dolor crónico ${ }^{[6]}$, así como un retorno más rápido a las actividades de la vida diaria ${ }^{[7]}$, también se asocia a mayores tasas de recurrencias ${ }^{[3]}$ y costos de implementación, lo que limita el acceso masivo a este tipo de tecnologías. Por esta razón, las técnicas de cirugía abierta aún representan las técnicas más utilizadas en el Perú ${ }^{[8]}$.

Contrario a lo que podría esperarse, si bien a la fecha se han descrito una gran número de técnicas de herniorrafía abierta, tampoco existe un consenso entre los cirujanos con respecto a cuál debería considerarse como la técnica quirúrgica de elección. Entre las principales técnicas de cirugía abierta para el manejo de hernias inguinales tenemos a las técnicas de abordaje anterior no protésicas (Bassini ${ }^{[9]}$, Halsted ${ }^{[10]}$, Shouldice ${ }^{[11]}$, Ferguson ${ }^{[12]}$, McVay ${ }^{[33]}$, Marcy ${ }^{[14]}$ y Nyhus-Condon $\left.{ }^{[15]}\right]$, las técnicas de abordaje anterior protésicas o con malla (Lichtenstein ${ }^{[16]}$, Trabucco ${ }^{[17]}$, Rutkow ${ }^{[18]}$, Flament ${ }^{[19]}$ y sus variantes Valenti ${ }^{[20]}$ y Donati ${ }^{[21]}$ ) y las técnicas de abordaje posterior (Nyhus ${ }^{[22]}$, Read ${ }^{[23]}$, Rives ${ }^{[24]}$, Wantz ${ }^{[25]}$, Stoppa ${ }^{[26]}$, Kugel $\left.^{[27]}\right)^{[28]}$. De éstas, la técnica quirúrgica más practicada en nuestro medio es la técnica libre de tensión, más conocida como la técnica de Lichtenstein.

La técnica Lichtenstein tiene como primer paso la identificación de la hernia inguinal, las estructuras nerviosas, vasos sanguíneos y cordón espermático a partir de una disección extensa del conducto inguinal; como segundo paso, la reducción de la hernia con una sutura en bolsa de tabaco (si son pequeñas) o con un punto de surget continuo de la fascia transversalis (cuando es directa), con o sin la resección del saco indirecto; y como tercer paso, la colocación de una malla (generalmente de polipropileno) fijándola con suturas al ligamento inguinal y los tejidos circundantes asegurándose de superponerla al tubérculo púbico medial ${ }^{[16]}$. Lamentablemente, debido a la gran manipulación de las estructuras nerviosas durante la cirugía, la técnica de Lichtenstein ha sido asociada con una alta incidencia de complicaciones tales como dolor crónico y adormecimiento persistente. Como una alternativa, precisamente para evitar este tipo de complicaciones, se ha propuesto a la técnica de Nyhus, técnica en la que la hernia inguinal es reparada mediante la reconstrucción de la pared posterior del conducto inguinal con un abordaje posterior preperitoneal y la implantación de una malla protésica ${ }^{[22]}$.

El dolor crónico representa una complicación, largamente ignorada, del uso de la técnica de Lichtenstein ${ }^{[29]}$. Según la literatura la incidencia de esta complicación varía entre el 11\% ${ }^{[30]}$ y el $54 \%{ }^{[31]}$ de los casos. Este dolor crónico puede deberse a la inflamación producida por la sobre-manipulación de uno de los tres nervios (nervio íleo-hipogástrico, el nervio íleo-inguinal, y la rama genital del nervio génito-femoral) que transcurren por el conducto inguinal o, mucho más comúnmente, tratarse de un dolor neuropático de tipo iatrogénico producido por la lesión o constricción de cualquiera de estos tres nervios en la sutura o en la malla ${ }^{[32]}$. Por todo ello, parece existir una necesidad 
palpable de una técnica quirúrgica que nos permita disminuir no sólo la amplia disección del canal inguinal, con una menor manipulación de los nervios inguinales, sino también minimizar la interacción entre la malla y estructuras sensibles como el cordón espermático y los nervios periféricos ${ }^{[33]}$. De ahí que la técnica de Lichtenstein, que coloca la malla en un espacio avascular carente de estructuras nerviosas, fue propuesta como una alternativa más inocua a la técnica de Nyhus; sin embargo, la evidencia disponible no parece necesariamente respaldar esta hipótesis ${ }^{[34]}$.

Por todo lo anterior, el estudio tiene como objetivo comparar los beneficios y complicaciones tempranas y tardías del uso de la técnica de Nyhus como una alternativa a la técnica de Lichtenstein en el manejo de las hernias inguinales en una población con una alta incidencia de las mismas, como lo es la población sujeta de atención en el Hospital Militar Central (HMC).

\section{MATERIAL Y MÉTODOS}

\section{Diseño de estudio}

Se realizó un estudio trasversal, en el que se analizaron retrospectivamente los registros quirúrgicos e historias clínicas de todos los pacientes con hernia inguinal tratados quirúrgicamente en el HMC durante el período 2012-2014. El HMC es el hospital de referencia nacional del Ejército Peruano ubicado en Lima, Perú.

Esta información fue recogida y analizada con el objeto de determinar y comparar la incidencia de complicaciones tempranas (si se presentaron en los primeros tres meses del postquirúrgico) y tardías (si se presentaron entre los tres meses y los dos años del postquirúrgico) secundarias al uso de las técnicas de Lichtenstein y Nyhus en el manejo de hernias inguinales en el HMC. Adicionalmente, se analizó los potenciales factores predictores de estas complicaciones.

\section{Población y muestra}

La población de estudio la conformaron todos los pacientes con hernia inguinal tratados quirúrgicamente en el HMC durante el periodo Enero 2012-Diciembre del 2014. Según las estadísticas del servicio de cirugía general, durante el periodo de estudio, un total de 205 pacientes con hernia inguinal fueron tratados quirúrgicamente en el HMC. Para elegir a nuestra población objetivo se utilizó como criterio de inclusión todo paciente con diagnóstico de hernia inguinal tratado quirúrgicamente ya sea con la técnica de Lichtenstein o con la técnica de Nyhus en el HMC durante el período 2012-2014; mientras que se utilizó como criterios de exclusión el antecedente de cirugía abdominal y un riesgo quirúrgico alto. Dado que a priori se decidió incluir a todos los pacientes que cumplieran con estos criterios de selección no se previó realizar cálculo de tamaño de muestra alguno.

\section{Recolección de datos}

Las historias clínicas y registros quirúrgicos de todos los pacientes con diagnóstico de hernia inguinal que cumplieron con nuestros criterios de selección fueron revisadas a fin de levantar con precisión cada de las siguientes variables de interés del estudio: técnica quirúrgica, edad, sexo, índice de masa corporal, tipo de hernia, localización, clasificación de Nyhus, tiempo de enfermedad, tiempo quirúrgico, tipo de anestesia, tiempo de hospitalización postquirúrgico, complicaciones tempranas (neuralgia, seroma, edema escrotal, hematomas, muerte) y tardías (orquitis, atrofia testicular, dolor crónico, recurrencia o muerte). Todas estas variables fueron levantadas utilizando una lista de chequeo o Ficha de Recolección de Datos, la cual fue elaborada específicamente para facilitar un recojo completo, fidedigno y sin ningún identificador que permita reconocer las identidades de los participantes del estudio.

\section{Análisis de datos}

Primero se realizó un análisis descriptivo de los datos resumiendo las variables numéricas con media, desviación estándar, valores máximo y mínimo, previa comprobación de la distribución normal de estas variables con el test de Shapiro-Wilk; mientras que en el caso de las variables categóricas se utilizó su frecuencia absoluta y relativa. Después, se analizó la comparabilidad de ambos grupos haciendo uso de las pruebas $t$ de Student para la comparación de medias y la prueba exacta de Fisher para la comparación de proporciones. Para ello se cuantificó la incidencia de cualquier complicaciones, complicaciones tempranas y complicaciones tardías dicotomizando éstas como positivas (al menos una) o negativas (ninguna complicación). Posteriormente se realizó un análisis de factores asociados a la incidencia de cada una de este tipo de complicaciones utilizando un modelo de regresión logística y el método de modelos anidados considerando como cada una de las siguientes variables como potenciales factores asociados: género, edad, tiempo de enfermedad, localización de la hernia, tipo de hernia según la clasificación de Nyhus, índice de masa corporal (IMC), tiempo quirúrgico, tipo de anestesia, tiempo de hospitalización postquirúrgico. $Y$ finalmente se analizaron los factores asociados a la incidencia de complicaciones tempranas y tardías por separado utilizando el método forward y un modelo de regresión logística multivariante. En todos los casos el análisis de datos se realizó utilizando el paquete estadístico STATA ${ }^{\text {tm }}$ MP versión 13.0 (Stata Corp LP, College Station, Texas).

\section{Aspectos éticos}

En todos los casos los datos fueron levantados de manera anónima teniendo especial cuidado en que los datos no fueran identificables, otorgando a cada ficha de recolección de datos un código único que sólo fue manejado por los investigadores del estudio. El estudio fue revisado y aprobado por el Comité Institucional de Ética del HMC y por el comité científico de la Facultad de Medicina de la Universidad Nacional Mayor de San Marcos, pues fue la tesis de uno de los autores. 
Tabla 1. Características generales de los pacientes con hernia inguinal tratados quirúrgicamente.

\begin{tabular}{|c|c|c|c|c|c|c|}
\hline \multirow{2}{*}{ Características } & \multicolumn{2}{|c|}{ Lichtenstein } & \multicolumn{2}{|c|}{ Nyhus } & \multicolumn{2}{|c|}{ Total } \\
\hline & $n$ & $(\%)$ & $\mathrm{n}$ & $(\%)$ & $n$ & $(\%)$ \\
\hline Total de participantes & 180 & $(89,1)$ & 22 & $(10,9)$ & 202 & $(100,0)$ \\
\hline \multicolumn{7}{|l|}{ Género } \\
\hline Femenino & 64 & $(35,6)$ & 7 & $(31,8)$ & 71 & $(35,2)$ \\
\hline Masculino & 116 & $(64,4)$ & 15 & $(68,1)$ & 131 & $(64,8)$ \\
\hline \multicolumn{7}{|l|}{ Edad (años) } \\
\hline Media (DE) & 51,9 & $(18,1)$ & 56,7 & $(14,8)$ & 52,5 & 17,8 \\
\hline$<40$ & 50 & $(27,8)$ & 3 & $(13,6)$ & 53 & $(26,2)$ \\
\hline $40-59,9$ & 65 & $(36,1)$ & 10 & $(45,5)$ & 75 & $(37,1)$ \\
\hline$\geq 60$ & 65 & $(36,1)$ & 9 & $(41,0)$ & 74 & $(36,7)$ \\
\hline \multicolumn{7}{|l|}{ Tiempo de enfermedad (meses) } \\
\hline Media (DE) & 29,2 & $(22,1)$ & 21,8 & 11,1 & 18,4 & $(21,2)$ \\
\hline$<24$ & 70 & $(38,9)$ & 12 & $(54,6)$ & 82 & $(40,6)$ \\
\hline $24-47$ & 73 & $(40,6)$ & 7 & $(31,8)$ & 80 & $(39,6)$ \\
\hline$\geq 48$ & 37 & $(20,6)$ & 3 & $(13,6)$ & 40 & $(19,8)$ \\
\hline \multicolumn{7}{|l|}{ Tipo la hernia } \\
\hline Directa & 106 & $(58,9)$ & 14 & $(63,6)$ & 120 & $(59,4)$ \\
\hline Indirecta & 74 & $(41,1)$ & 8 & $(36,4)$ & 82 & $(40,6)$ \\
\hline \multicolumn{7}{|l|}{ Localización de la hernia } \\
\hline Izquierda & 134 & $(74,4)$ & 16 & $(72,7)$ & 150 & $(74,3)$ \\
\hline Derecha & 40 & $(22,2)$ & 5 & $(22,7)$ & 45 & $(22,3)$ \\
\hline Bilateral & 6 & $(3,3)$ & 1 & $(4,6)$ & 7 & $(3,7)$ \\
\hline \multicolumn{7}{|l|}{ NYHUS } \\
\hline Tipo II & 47 & $(24,8)$ & 3 & $(13,6)$ & 50 & $(24,8)$ \\
\hline Tipo III-A & 97 & $(56,9)$ & 18 & $(81,8)$ & 115 & $(56,9)$ \\
\hline Tipo III-B & 30 & $(15,4)$ & 1 & $(4,6)$ & 31 & $(15,4)$ \\
\hline Tipo IV & 6 & $(3,0)$ & 0 & $(0,0)$ & 6 & $(3,0)$ \\
\hline \multicolumn{7}{|l|}{$\mathrm{IMC}\left(\mathrm{kg} / \mathrm{m}^{2}\right)$} \\
\hline Media (DE) & 25,6 & $(2,1)$ & 26,0 & $(2,5)$ & 25,6 & $(2,2)$ \\
\hline$<25$ & 60 & $(33,3)$ & 7 & $(31,8)$ & 67 & $(33,2)$ \\
\hline $25-29.9$ & 113 & $(62,8)$ & 12 & $(54,6)$ & 125 & $(61,9)$ \\
\hline$\geq 30$ & 7 & $(3,9)$ & 3 & $(13,6)$ & 10 & $(4,9)$ \\
\hline \multicolumn{7}{|l|}{ Tiempo quirúrgico (minutos) } \\
\hline Media (DE) & 43,5 & $(13,5)$ & 39,1 & $(8,9)$ & 42,9 & 13,1 \\
\hline$\leq 30$ & 9 & $(5,0)$ & 4 & $(18,2)$ & 13 & $(6,4)$ \\
\hline $31-60$ & 137 & $(76,1)$ & 17 & $(77,3)$ & 154 & $(76,2)$ \\
\hline$\geq 60$ & 34 & $(18,9)$ & 1 & $(4,6)$ & 35 & $(17,3)$ \\
\hline \multicolumn{7}{|l|}{ Tiempo de hospitalización (días) } \\
\hline Media (DE) & 3,9 & $(1,2)$ & 3,8 & $(0,8)$ & 3,9 & $(1,1)$ \\
\hline$<3$ & 8 & $(4,4)$ & 1 & $(4,6)$ & 9 & $(4,5)$ \\
\hline $3-4$ & 131 & $(72,8)$ & 17 & $(77,3)$ & 148 & $(73,2)$ \\
\hline $5-6$ & 31 & $(17,2)$ & 4 & $(18,2)$ & 35 & $(17,3)$ \\
\hline$\geq 6$ & 10 & $(5,6)$ & 0 & $(0,0)$ & 10 & $(4,9)$ \\
\hline
\end{tabular}

$D E$ : Desviación estándar; IMC: Índice de masa corporal. 


\section{RESULTADOS}

\section{Características de la población de estudio}

Durante el periodo enero 2012-diciembre 2014, un total de 205 pacientes con hernia inguinal fueron tratados quirúrgicamente, de cuales tres no pudieron ser analizados al no encontrarse sus registros quirúrgicos $(n=1)$ o historias clínicas $(n=2)$, no excluyéndose ningún caso por los criterios de exclusión originalmente previstos. De los 202 casos analizados, la mayoría (65\%) eran varones $y$, en general, con una edad promedio de 52,5 $\pm 17,8$ años (rango: 22-87 años). Clínicamente se encontró un tiempo de enfermedad promedio de $28,4 \pm 21,2$ meses, siendo los tipos de hernias inguinales más frecuentes las de tipo directa (59\%), NYHUS III-A (57\%) y las de localización izquierda (74\%). Adicionalmente, se encontró que la mayoría de los pacientes padecía de sobrepeso u obesidad (67\%), registrando un IMC promedio de $25,6 \pm 2,2 \mathrm{~kg} / \mathrm{m}^{2}$.

En promedio las cirugías duraron 52,4 \pm 13,9 minutos permaneciendo luego hospitalizados por $3,9 \pm 1,1$ días. Del total de herniorrafías, 180 (89\%) fueron practicadas con la técnica de Lichtenstein mientras que 22 (11\%) fueron practicadas con la técnica de Nyhus. Al comparar ambos grupos no se encontraron diferencias estadísticamente significativas $(p<0,05)$ en ninguno de las características generales estudiadas (Tabla 1).

\section{Incidencia de complicaciones}

Al analizar la incidencia de complicaciones se encontró una tasa de letalidad del $0 \%$ y una incidencia de complicaciones en general del $18 \%$, una incidencia de complicaciones tempranas del $16 \%$ y una incidencia de complicaciones tardías del $4 \%$. De estas, las más complicaciones tempranas y tardías más frecuentes fueron las neuralgias (12\%) y las recurrencias (3\%), respectivamente. Al comparar ambas técnicas quirúrgicas se encontró diferencias significativas tanto en la incidencia de complicaciones en general ( $20 \%$ vs. $0 \% ; p=0,016)$ como en la incidencia de complicaciones tempranas ( $17 \%$ vs. $0 \%, p=0,028)$. Sin embargo, a pesar de no encontrarse complicaciones tardías en el caso de los pacientes intervenidos con la técnica de Nyhus no se encontraron diferencias en la incidencia de complicaciones tardías entre ambas técnicas ( $4 \%$ vs. $0 \%, p=0,603$ ). De la misma manera no se encontraron diferencias estadísticamente significativas $(p>0,050)$ cuando se comparó la incidencia de cada complicación temprana o tardía encontrada por separado (Tabla 2).

\section{Análisis de regresión}

Al analizar cada una de las diferentes características generales (Tabla 3), clínicas o quirúrgicas, como potenciales factores asociados se encontró primero que la técnica de Lichtenstein predecía el 100\% de las complicaciones post-quirúrgicas, tanto tempranas como tardías. Lamentablemente por lo mismo,

Tabla 2. Características generales de los pacientes con hernia inguinal tratados quirúrgicamente.

\begin{tabular}{|c|c|c|c|}
\hline Características & $\begin{array}{l}\text { Lichtenstein } \\
\mathbf{N}(\%)\end{array}$ & $\begin{array}{l}\text { Nyhus } \\
\text { N(\%) }\end{array}$ & $\begin{array}{l}\text { Total } \\
\text { N }(\%)\end{array}$ \\
\hline \multicolumn{4}{|l|}{ Complicaciones } \\
\hline Negativo & $144(80,0)$ & $22(100,0)$ & $170(82,2)$ \\
\hline Positivo & $36(20,0) *$ & $0(0,0)$ & $36(17,8)$ \\
\hline Solo una & $32(13,9)$ & $0(0,0)$ & $32(15,8)$ \\
\hline Una o más & $4(3,9)$ & $0(0,0)$ & $4(2,0)$ \\
\hline \multicolumn{4}{|l|}{ Complicaciones tempranas } \\
\hline Negativo & $148(82,2)$ & $22(100,0)$ & $170(84,2)$ \\
\hline Positivo & $32(17,8) *$ & $0(0,0)$ & $32(15,8)$ \\
\hline Neuralgia & $25(13,9)$ & $0(0,0)$ & $25(12,4)$ \\
\hline Seroma & $7(3,9)$ & $0(0,0)$ & $7(3,5)$ \\
\hline \multicolumn{4}{|l|}{ Complicaciones tardías } \\
\hline Negativo & $172(95,6)$ & $22(100,0)$ & $172(95,6)$ \\
\hline Positivo & $8(4,4)$ & $0(0,0)$ & $8(4,4)$ \\
\hline Recurrencia de hernia & $5(2,8)$ & $0(0,0)$ & $5(2,5)$ \\
\hline Orquitis & $2(1,1)$ & $0(0,0)$ & $2(0,9)$ \\
\hline Dolor crónico & $1(0,5)$ & $0(0,0)$ & $1(0,5)$ \\
\hline
\end{tabular}

$* p<0,050$ 
Tabla 3. Análisis de regresión.

\begin{tabular}{|c|c|c|c|}
\hline Modelo & OR & IC $95 \%$ & Valor $p$ \\
\hline \multicolumn{4}{|l|}{ Complicaciones } \\
\hline Tiempo quirúrgico (min.) & 1,09 & $1,05-1,13$ & $<0,001$ \\
\hline Tiempo de hospitalización (días) & 1,61 & $1,14-2,28$ & 0,007 \\
\hline \multicolumn{4}{|l|}{ Complicaciones post Lichtenstein * } \\
\hline Tiempo quirúrgico (min.) & 1,09 & $1,05-1,12$ & $<0,001$ \\
\hline Tiempo de hospitalización (días) & 1,61 & $1,14-2,27$ & 0,007 \\
\hline \multicolumn{4}{|l|}{ Complicaciones tempranas } \\
\hline Tiempo quirúrgico (min.) & 1,07 & $1,05-1,11$ & $<0,001$ \\
\hline Tiempo de hospitalización (días) & 1,55 & $1,10-2,19$ & 0,013 \\
\hline \multicolumn{4}{|c|}{ Complicaciones tempranas post Lichtenstein * } \\
\hline Tiempo quirúrgico (min.) & 1,07 & $1,04-1,10$ & $<0,001$ \\
\hline Tiempo de hospitalización (días) & 1,54 & $1,09-2,17$ & 0,013 \\
\hline \multicolumn{4}{|l|}{ Complicaciones tardías } \\
\hline Tiempo quirúrgico (min.) & 1,09 & $1,04-1,13$ & $<0,001$ \\
\hline \multicolumn{4}{|c|}{ Complicaciones tardías post Lichtenstein * } \\
\hline Tiempo quirúrgico (min.) & 1,08 & $1,03-1,13$ & 0,001 \\
\hline
\end{tabular}

OR: odds ratio; IC 95\%, Intervalo de confianza al 95\%

* Análisis de regresión realizado sólo entre los pacientes operados con la técnica de Lichtenstein.

no se puedo estimar la magnitud de asociación entre este factor y las variables desenlace de interés de este estudio. Independientemente de ello en el análisis de regresión multivariante se encontró que el principal predictor de complicaciones tanto en general (Odds ratio [OR]: 1,09; Intervalo de confianza [IC] al 95\%: 1,05-1,13; $p<0,001)$, como tempranas (OR: 1,07; IC 95\%: 1,05-1,11; $p<0,001$ ) y tardías (OR: 1,61; IC 95\%: $1,14-2,28 ; p<0,001)$ es el tiempo quirúrgico, incrementándose el riesgo de estas complicaciones con cada minuto adicional del tiempo quirúrgico. Adicionalmente, se encontró que la incidencia de complicaciones en general (OR: 1,61 ; IC 95\%: $1,14-2,28$ ) y de las complicaciones tempranas (OR: 1,55 ; IC 95\%: 1,10-2,19) se incrementaban también mientras mayor el tiempo de hospitalización. Finalmente, tal como se puede apreciar en la Tabla 3, se encontró que las magnitudes de asociación previamente descritas variaron mínimamente $(<1 \%)$ cuando se replicó el análisis de regresión multivariante solo en la población de pacientes operados con la técnica de Lichtenstein, lo que permite inferir que la técnica quirúrgica no se comportaban con un factor confusor o modificador de efecto de las asociaciones encontradas en nuestro modelo de regresión.

\section{DISCUSIÓN}

En la experiencia del HMC, tanto la técnica de Lichtenstein como Nyhus representan técnicas de herniorrafía abierta seguras para el manejo quirúrgico de las hernias inguinales dado que ambas fueron asociadas con tasas de letalidad de $0 \%$ y tasas de complicaciones en general inferiores al $20 \%$, siendo más del $80 \%$ de las mismas reportadas de ocurrencia temprana (dentro de los primeros tres meses del postquirúrgico). Sin embargo, al comparar la seguridad de ambas técnicas se encontró que tanto las complicaciones en general como las complicaciones tempranas fueron significativamente más frecuentes con la técnica de Lichtenstein que con la técnica de Nyhus, incrementándose en ambos casos mientras mayor el tiempo quirúrgico y/o el tiempo de hospitalización. De manera general, se encontró que el predictor más importante de complicaciones postquirúrgicas, tanto tempranas como tardías, es el tiempo quirúrgico. Adicionalmente, se encontró que el tiempo de hospitalización también se comportaba como un predictor importante de la incidencia de complicaciones pero solo en el caso de las complicaciones en general y de las complicaciones tempranas. De esta manera, independientemente de la técnica utilizada, mientras mayor el tiempo quirúrgico o mayor el tiempo de hospitalización mayor la incidencia de complicaciones general y mayor la incidencia de complicaciones tempranas tales como neuralgias y seromas.

Los resultados de este estudio dan cuenta que, en la experiencia del $\mathrm{HMC}$, se encontraron diferencias significativas en la incidencia de complicaciones postquirúrgicas entre las técnicas de Lichtenstein y Nyhus. Específicamente, se encontró que de cada 20 pacientes con hernias inguinales tratados quirúrgicamente con la técnica de Lichtenstein aproximadamente cuatro (18\%) reportaron complicaciones de tipo tempranas ( $14 \%$ neuralgia y $4 \%$ seromas) y 
aproximadamente uno (4\%) reportaron complicaciones tardías (3\% recurrencias y $1 \%$ orquitis); mientras que de 20 pacientes tratados con la técnica de Nyhus ninguno presentó estas complicaciones. Entre las muchas razones que podrían explicar este hallazgo, la primera que debemos considerar es que efectivamente la técnica de Nyhus se asocia con menos complicaciones que la técnica de Lichtenstein, sobre todo tomando en consideración cuando que más de las dos terceras partes (69\%) de estas complicaciones fueron del tipo neuralgia $u$ otros dolores asociados. Esto es de esperarse ya que una de las principales ventajas que ofrece la técnica de Nyhus por sobre la técnica de Lichtenstein es que evita la manipulación de las estructuras nerviosas que transcurren por el canal inguinal, las cuales incluyen el nervio iliohipogástrico, el nervio ilioinguinal y la rama genital del nervio génito-femoral. De hecho, nuestros resultados en general coinciden con lo recientemente reportado porYang et al. quienes luego de seguir un total de 107 casos de herniorrafía con la técnica de Nyhus por un promedio de 42 meses encontraron como complicaciones posquirúrgicas solo dos (2\%) casos de seromas y siete $(7 \%)$ casos de complicaciones periquirúrgicas, incluyendo dos (2\%) casos de sangrados menores en el intraoperatorio, dos (2\%) de infecciones de herida operatorio en el intrahospitalario, y tres $(3 \%)$ casos de retención urinaria post anestesia regional ${ }^{[35]}$.

Otra explicación para las diferencias encontradas deviene de la posibilidad de un sesgo del operador, es decir, que las diferencias se expliquen por el hecho de que la gran mayoría de cirujanos son entrenados en el dominio de la técnica de Lichtenstein pero solo algunos, por lo general los más experimentados, son también entrenados en el dominio de la técnica de Nyhus. De hecho, dado la experiencia del cirujano ha sido consistentemente reportada como un importante predictor del éxito de las herniorrafías ${ }^{\left[{ }^{66]}\right.}$, el hecho de que solo cirujanos experimentados practican las herniorrafías con la técnica de Nyhus en el HMC también podría explicar las diferencias encontradas en los tiempos quirúrgicos y en la incidencia de complicaciones a favor de la técnica de Nyhus comparada con la técnica de Lichtenstein. Específicamente, y a diferencia de lo reportado por otros estudios como el de Liu et $a l{ }^{[37]}$, en el nuestro no solo no se encontraron diferencias en los tiempos quirúrgicos de ambas técnicas sino que en ambos casos los tiempos quirúrgicos se encontraron por debajo de los reportados por otros investigadores. Por ejemplo, según Liu et al. ${ }^{[37]}$, la técnica Nyhus es una técnica significativamente más rápida que la técnica de Lichtenstein $(60 \pm 11$ minutos vs. $63 \pm 8$ minutos; $p=0,032$ ), mientras que en nuestro estudio no encontramos mayores diferencias entre ambas técnicas $(43,0 \pm 13,6$ minutos vs. $39,1 \pm 8,9$ minutos; $p=0,140)$.

Por otro lado, también cabe la posibilidad de que las diferencias encontradas se deban que nuestro estudio pudo haber carecido de potencia suficiente para detectar complicaciones secundarias a la técnica de Nyhus o que se haya producido un subregistro de complicaciones en las cirugías practicadas con la técnica de Nyhus, aunque ambas posibilidades sean remotas ya para invalidar dicho hallazgo tendrían que haberse solo en un brazo del estudio y no en los dos. Por el contrario, un dato que refuerza la validez de nuestros hallazgos, es el hecho de que la incidencia de recurrencias, seroma y orquitis isquémica encontrada entre los pacientes operados con la técnica de Lichtenstein (3\%) se acerca mucho a lo reportado en la literatura. Según la revisión sistemática de McCormack et al ${ }^{[38]}$, quienes analizaron un total de 6642 cirugía de Lichtenstein, 3138 laparoscópicas y 3504 abiertas, la incidencia de recurrencias esperada en el uso de esta técnica se estima en $\sim 2,7 \%$ y $\sim 3,1 \%$, respectivamente, tasa muy similar a la encontrada en nuestro estudio. Según Matthews la incidencia de seroma $u$ orquitis en pacientes operados con la técnica de Lichtenstein $(n, 965)$ hasta los dos años del seguimiento se encuentra por debajo del $4 \%$ y $2 \%$, respectivamente ${ }^{[39]}$. Más aún de acuerdo con uno de los pocos ensayos clínicos disponibles la incidencia de dolor crónico (dolor persistente por más dos meses) se estimó en un $6 \%{ }^{[40]}$.

Un hallazgo de nuestro estudio que bien vale la pena destacar constituye la identificación del tiempo quirúrgico y al tiempo de hospitalización como los principales factores predictores de la ocurrencia de complicaciones post herniorrafía, independientemente de la técnica quirúrgica utilizada. De acuerdo con nuestros datos las chances de que ocurra una complicación se incrementan significativamente con cada minuto de cirugía y con cada día extra que el paciente permanece hospitalizado. Sin embargo, y probablemente por su mayor variabilidad y capacidad discriminatoria, cuando se realizó el análisis diferenciado se encontró que el tiempo quirúrgico constituía el principal predictor tanto de las complicaciones tempranas como de las complicaciones tardías. Adicionalmente, se encontró que la incidencia de complicaciones tempranas también se incrementaba mientras mayor el tiempo de hospitalización. Lamentablemente luego de una amplia búsqueda de referencias no encontramos antecedentes con los cuales contrastar este hallazgo por lo que al tratarse de un hallazgo original bien vale la pena que sea explorado en estudios subsiguientes ya con un diseño prospectivo y un mayor tamaño de muestra.

Con respecto a las limitaciones de nuestro estudio, tomando en consideración las características de su diseño, creemos pertinente discutir por lo menos las siguientes tres: Primero, que pese a haber incluido todos los casos elegibles durante el periodo de interés el tamaño de la muestra de casos analizada es pequeña, particularmente en el caso de las herniorrafías practicadas con la técnica de Nyhus. Esto si bien pudo haber limitado la validez externa del estudio, lo cierto es que el estudio contó con un poder de estudio suficiente como para detectar diferencias clínicamente relevantes y estadísticamente significativas. Segundo, que tratándose de un estudio retrospectivo con una validez interna relativamente limitada, lo cierto es que en el caso de las complicaciones de interés las mismas se encontraban rigurosamente documentadas y, si existió algún subregistro, es improbable que mismo haya sido de tipo diferencial (entiéndase, haber afectado, en mayor o menor medida a uno de las técnicas estudiadas). Y tercero, si bien es muy probable que existan otros confusores no medidos en nuestro análisis, también es poco factible que el efecto de 
los mismos haya pasado desapercibido en nuestro análisis de regresión multivariante y que, de haber sido incluido, cambien radicalmente los resultados de nuestro análisis, aunque esa remota posibilidad siempre exista.

Finalmente, al momento de interpretar nuestros resultados es importante tomar en consideración el perfil clínico epidemiológico de nuestra población de estudio, el cual se caracterizó por tratarse predominantemente de pacientes adultos, varones, con una hernia inguinal de tipo directa, de localización izquierda y de tipo Nyhus IIIA, con un tiempo de enfermedad prolongado. Adicionalmente, es importante tomar en consideración que si bien el HMC atiende a pacientes particulares la gran mayoría de pacientes atendidos son personal militar y sus familiares, por lo que la gran mayoría gozan de algún nivel de cobertura de sus gastos de atención.

En conclusión, las técnicas de Lichtenstein y Nyhus demostraron ser técnicas bastante seguras en el manejo quirúrgico de las hernias inguinales asociándose en ambos casos a niveles bajos de complicaciones tempranas y tardías, aunque las complicaciones tempranas tendieron a incrementarse, independientemente de la técnica utilizada, mientras mayor fue el tiempo quirúrgico y el tiempo de hospitalización.

\section{Fuente de financiamiento:}

Los autores declaran no haber recibido ninguna financiación para la realización de este trabajo.

\section{Declaración de conflicto de intereses:}

Los autores declaran no tener conflicto de intereses con la publicación de este artículo.

\section{REFERENCIAS BIBLIOGRÁFICAS}

1. Eurostat [página en Internet]. Surgical operations and procedures statistics. Further Eurostat information. European Comission Authentication Services (ECAS); 2016 [citado el 17 de octubre de 16]. Disponible en: http://ec.europa.eu/eurostat/statisticsexplained/index.php/Surgical_operations_and_procedures_ statistics - Further_Eurostat_information

2. Memon MA, Cooper NJ, Memon B, Memon MI, Abrams KR Meta-analysis of randomized clinical trials comparing open and laparoscopic inguinal hernia repair. Br J Surg. 2003;90(12):1479-92.

3. Neumayer L, Giobbie-Hurder A, Jonasson O, Fitzgibbons R, Jr., Dunlop D, Gibbs J, et al. Open mesh versus laparoscopic mesh repair of inguinal hernia. N Engl J Med. 2004;350(18):1819-27.

4. Dedemadi G, Sgourakis G, Radtke A, Dounavis A, Gockel I, Fouzas I, et al. Laparoscopic versus open mesh repair for recurrent inguinal hernia: a meta-analysis of outcomes. Am J Surg. 2010;200(2):291-7.

5. O'Reilly EA, Burke JP, O'Connell PR. A meta-analysis of surgical morbidity and recurrence after laparoscopic and open repair of primary unilateral inguinal hernia. Ann Surg. 2012;255(5):846-53.

6. Niccolai $\mathrm{P}$, Ouchchane $\mathrm{L}$, Libier $\mathrm{M}$, Beouche $\mathrm{F}$, Belon $\mathrm{M}$, Vedrinne $\mathrm{JM}$, et al. Persistent neuropathic pain after inguinal herniorrhaphy depending on the procedure (open mesh v. laparoscopy): a propensity-matched analysis. Can J Surg. 2015;58(2):114-20.
7. Pisanu A, Podda M, Saba A, Porceddu G, Uccheddu A. Metaanalysis and review of prospective randomized trials comparing laparoscopic and Lichtenstein techniques in recurrent inguinal hernia repair. Hernia. 2015;19(3):355-66.

8. Weber Sanchez A, Vásquez JA, Mansilla Doria P, Cueto Garcia J. La hernia inguinal en la era laparoscópica. Rev Med Hered. 2009;10(1):9-18.

9. Elsebae MM, Nasr M, Said M. Tension-free repair versus Bassini technique for strangulated inguinal hernia: A controlled randomized study. Int J Surg. 2008;6(4):302-5.

10. Halsted WS. III. The radical cure of inguinal hernia in the male. Ann Surg. 1893;17(5):542-56.

11. Shouldice EB. The Shouldice repair for groin hernias. Surg Clin North Am. 2003;83(5):1163-87, vii.

12. Ferguson $A H$. VI. On the radical cure of inguinal and femoral hernia by operation. Ann Surg. 1895;21(5):547-64.

13. MCVAY CB. Inguinal and femoral hernioplasty; anatomic repair. Arch Surg. 1948;57(4):524-30.

14. Griffith CA. The Marcy repair of indirect inguinal hernia. Surg Clin North Am. 1971;51(6):1309-16.

15. Nyhus LM, Condon RE, Harkins HN. Clinical experiences with preperitoneal hernial repair for all types of hernia of the groin, with particular reference to the importance of transversalis fascia analogues. Am J Surg. 1960;100:234-44.

16. Lichtenstein IL, Shulman AG, Amid PK, Montllor MM. The tensionfree hernioplasty. Am J Surg. 1989;157(2):188-93.

17. Trabucco EE. The office hernioplasty and the Trabucco repair. Ann Ital Chir. 1993;64(2):127-49.

18. Robbins AW, Rutkow IM. The mesh-plug hernioplasty. Surg Clin North Am. 1993;73(3):501-12.

19. Lardennois B, Benoist M, Hibon J, Flament JB. [Results in the use of dacron material in the treatment of large eviscerations with respect to 37 cases]. Acta Chir Belg. 1971;70(3):287-90. [Article in French]

20. Valenti G, Testa A, Capuano G. [The dynamic self-regulating prosthesis. A new method for the treatment of hernias in the inguinal area: preliminary notes on 153 cases]. Minerva Chir. 1997;52(10):1247-53. [Article in Italian]

21. Donati $A, Z$ Zanghi $G$, Brancato $G$, Privitera A, Donati M. [Hernia repair with local anesthesia]. Ann Ital Chir. 1999;70(5):723-8. [Article in Italian]

22. Patino JF, Garcia-Herreros LG, Zundel N. Inguinal hernia repair. The Nyhus posterior preperitoneal operation. Surg Clin North Am. 1998;78(6):1063-74.

23. Read RC. Pre-extraperitoneal approach to inguinofemoral herniorrhaphy. Am J Surg. 1967;114(5):672-8.

24. Rives J. Surgical treatment of the inguinal hernia with dacron patch. Int Surg. 1967;47(4):360-1.

25. Wantz GE. [Technique of properitoneal hernioplasty. Unilateral reinforcement of the visceral sac with Mersilene giant prosthesis]. Chirurgie. 1993;119(6-7):321-6. [Article in French]

26. Champault GG, Rizk N, Catheline JM, Turner R, Boutelier P. Inguinal hernia repair: totally preperitoneal laparoscopic approach versus Stoppa operation: randomized trial of 100 cases. Surg Laparosc Endosc. 1997;7(6):445-50.

27. Kugel RD. Minimally invasive, nonlaparoscopic, preperitoneal, and sutureless, inguinal herniorrhaphy. Am J Surg. 1999;178(4):298-302. 
28. Basile F, Biondi A, Donati M. Surgical approach to abdominal wall defects: history and new trends. Int J Surg. 2013;11 Suppl 1:S20-3.

29. Kalliomaki ML, Sandblom G, Gunnarsson U, Gordh T. Persistent pain after groin hernia surgery: a qualitative analysis of pain and its consequences for quality of life. Acta Anaesthesiol Scand. 2009; $53(2): 236-46$.

30. Nienhuijs S, Staal E, Keemers-Gels M, Rosman C, Strobbe L. Pain after open preperitoneal repair versus Lichtenstein repair: a randomized trial. World J Surg. 2007;31(9):1751-7.

31. PoobalanAS, Bruce J, SmithWC, King PM, KrukowskiZH, Chambers WA. A review of chronic pain after inguinal herniorrhaphy. Clin J Pain. 2003;19(1):48-54.

32. Kehlet $H$, Bay-Nielsen M, Danish Hernia Database C. Nationwide quality improvement of groin hernia repair from the Danish Hernia Database of 87,840 patients from 1998 to 2005 . Hernia. 2008;12(1):1-7.

33. Gunal O, Ozer S, GurleyikE, BahcebasiT. Does the approach to the groin make a difference in hernia repair? Hernia. 2007;11(5):429-34.

34. Vatansev C, Belviranli M, Aksoy F, Tuncer S, Sahin M, Karahan O. The effects of different hernia repair methods on postoperative pain medication and CRP levels. Surg Laparosc Endosc Percutan Tech. 2002;12(4):243-6.
35. Yang B, Jiang ZP, Li YR, Zong Z, Chen S. Long-term outcome for open preperitoneal mesh repair of recurrent inguinal hernia. Int J Surg. 2015;19(1):134-6.

36. Treadwell J, Tipton K, Oyesanmi O, Sun F, Schoelles K. Surgical options for inguinal hernia: comparative effectiveness review. Rockville: Agency for Healthcare Research and Quality (US); 2012.

37. Liu Z, Sun M, Zhang L, Wu W, Wang W, Li X, et al. [Comparison of open preperitoneal repair and Lichtenstein herniorraphy on the surgical treatment of inguinal hernia]. Zhonghua Wai Ke Za Zhi. 2014;52(9):682-5. [Article in Chinese]

38. McCormack K, Scott NW, Go PM, Ross S, Grant AM; EU Hernia Trialists Collaboration. Laparoscopic techniques versus open techniques for inguinal hernia repair. Cochrane Database Syst Rev. 2003;(1):CDo01785.

39. Matthews RD, Anthony T, Kim LT, Wang J, Fitzgibbons RJ Jr, Giobbie-Hurder A, et al. Factors associated with postoperative complications and hernia recurrence for patients undergoing inguinal hernia repair: a report from the VA Cooperative Hernia Study Group. Am J Surg. 2007;194(5):611-7.

40. Erhan Y, Erhan E, Aydede H, Mercan M, Tok D. Chronic pain after Lichtenstein and preperitoneal (posterior) hernia repair. Can J Surg. 2008;51(5):383-7.

\title{
Las ediciones anteriores de Acta Médica Peruana están disponibles en:
}

\author{
www.scielo.org.pe
}

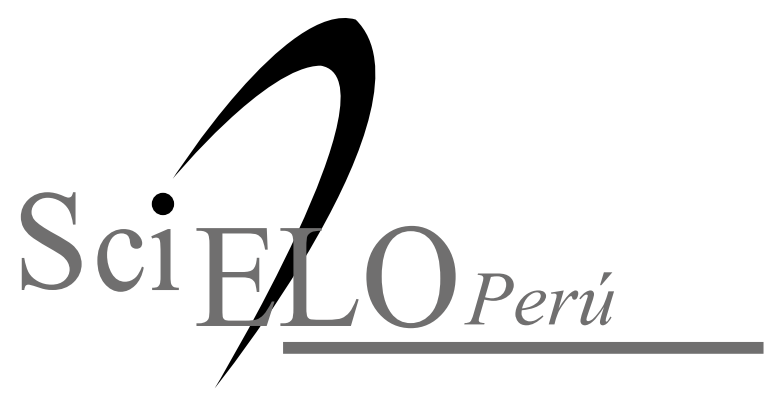

